\title{
Heart Rhythm Disturbances Associated With Rupatadine: A Case Series From the Spanish and Portuguese Pharmacovigilance Systems
}

\author{
A Carvajal $^{1}$, D Macíass ${ }^{1}$, I Salado ${ }^{1}$, M Sáinz $^{1}$, S Ortega $^{1}$, C Campo $^{1}$, J García del Pozo ${ }^{1}$, LH Martín Arias $^{1}$, \\ A Velasco $^{1}$, S Gonçalves ${ }^{2}$, R Pombal ${ }^{2}$ and R Carmona ${ }^{2}$
}

We searched the Spanish and Portuguese pharmacovigilance databases for spontaneous case reports of heart rhythm disturbances associated with rupatadine and other new $\mathrm{H} 1$ antihistamines. Five cases were found involving patients treated with rupatadine (13.9\% of all reports relating to this drug). In all five cases, the reaction started after exposure and resolved when the drug was discontinued. In two cases, rupatadine was the only medication being taken by the patient, and no other condition that could explain the heart rhythm disturbances was diagnosed. The reporting odds ratio was 3.2 (95\% confidence interval, 1.0-10.5). The reporting rate was 2 cases per 100,000 patients treated per year (95\% confidence interval, $0.4-6.0)$. These results suggest a causal relationship between rupatadine and heart rhythm disturbances.

Since the mid-1980s, a number of histamine H1 receptor antagonists (antihistamines) have been introduced onto the market worldwide. These second-generation antihistamines have fewer sedating effects than older antihistamines. All have similar chemical structures. Some are active metabolites of other antihistamine compounds; for example, fexofenadine, levocetirizine, and desloratadine are active metabolites of terfenadine, cetirizine, and loratadine, respectively. Rupatadine, introduced on 1 March 2003, inhibits both peripheral H1 histamine receptors and the platelet-activation factor. This may explain why rupatadine is more effective in treating allergic disorders than antihistamines that do not inhibit the plateletactivation factor. ${ }^{1,2}$

Most of the antihistamines in use have been associated with cardiotoxicity. ${ }^{3,4}$ Astemizole and terfenadine were removed from the market for this reason. ${ }^{5,6}$ Some of the newer antihistamines have been termed "third generation" because they are thought to have no cardiotoxic effect. ${ }^{7}$

In 2007, our pharmacovigilance center in Valladolid had knowledge of some cases, reported to the Spanish pharmacovigilance system, of heart rhythm disturbance presumably associated with rupatadine. This prompted us to investigate the possibility of a causal relationship between heart rhythm disturbances and rupatadine and other new antihistamines using data from the Portuguese and Spanish pharmacovigilance systems.

\section{RESULTS}

From 1 March 2003 until 12 December 2007, 36 reports in which rupatadine was associated with adverse reactions were collected by the Spanish $(n=32)$ and Portuguese $(n=4)$ pharmacovigilance systems. Five of these reports (13.9\%) related to heart rhythm disturbances. Table 1 summarizes these five cases, which occurred in four men and one woman. For four patients, the dose of rupatadine was $10 \mathrm{mg} /$ day; one patient received $20 \mathrm{mg} /$ day. The time between the patient first taking the drug and the onset of heart rhythm disturbances ranged from 1 day to 1 year. In three cases, rupatadine was the only drug the patient was taking; patients 2 and 5 were also being treated for other conditions. In addition to heart palpitations, patient 4 developed dry mouth, malaise, nausea, sweating, swelling, and weakness.

The estimated strength of association between heart rhythm disturbances of any type and rupatadine, astemizole, desloratadine, ebastine, loratadine, terfenadine, and levocetirizine was statistically significant (Table 2). The reported rates of heart rhythm disturbances for various $\mathrm{H} 1$ antihistamines are shown in Table 3. For azelastine and fexofenadine, there were no cases of

${ }^{1}$ Instituto de Farmacoepidemiología, Universidad de Valladolid, Valladolid, Spain; ${ }^{2}$ INFARMED Autoridade Nacional do Medicamento e Produtos de Saúde, I.P., Lisbon, Portugal. Correspondence: A Carvajal (carvajal@ife.uva.es) 
Table 1 Case series of heart rhythm disturbances associated with rupatadine reported to the Spanish and Portuguese pharmacovigilance systems

\begin{tabular}{|c|c|c|c|c|c|c|c|}
\hline Case & Age (years)/sex & Heart rhythm disturbance & $\begin{array}{l}\text { Induction } \\
\text { period (days) }\end{array}$ & $\begin{array}{l}\text { Duration } \\
\text { (days) }\end{array}$ & Other drugs or conditions & Indication & Outcome \\
\hline \multicolumn{8}{|c|}{ Spanish Pharmacovigilance System } \\
\hline 1 & 69/Male & Ventricular tachycardia & 365 & 3 & Coronary ischemia & Allergy & Recovered \\
\hline 2 & 73/Male & Long QT/torsade de pointes & 14 & 2 & Sertraline $^{a}$ & Allergic rhinitis & $\begin{array}{l}\text { Recovered/ } \\
\text { sequelae }\end{array}$ \\
\hline 3 & 30/Female & Tachycardia/palpitations & ND & 7 & - & Not stated & Recovered \\
\hline \multicolumn{8}{|c|}{ Portuguese Pharmacovigilance System } \\
\hline 4 & 32/Male & Palpitations & Months & ND & - & Eczema & Recovered \\
\hline 5 & 58/Male & Extrasystoles & 3 & ND & $\begin{array}{l}\text { Prostatic hyperplasia; aspirin, } \\
\text { serenoa repens, atorvastatin }\end{array}$ & Rhinitis & Recovered \\
\hline
\end{tabular}

Total number of reports from Spain for rupatadine was 32 during the period studied; from Portugal the corresponding figure was 4.

ND, no data.

apatient with depression, diabetes, hypertension, and prostate cancer, also treated with amlodipine, glimepiride, aspirin, chlortalidone, ezetimibe, insulin, pentoxifilin, lormetazepam, omeprazol, and eprosartan. The patient had several episodes of syncope.

Table 2 Rupatadine and heart rhythm disturbances

\begin{tabular}{lcc}
\hline Antihistamine & Heart rhythm disturbances (\%) & ROR \\
\hline Astemizole & $8(8.3)$ & $2.8(1.4-5.9)$ \\
\hline Azelastine & $1(3.5)$ & $1.1(0.1-7.9)$ \\
\hline Cetirizine & $8(3.6)$ & $1.1(0.6-2.3)$ \\
\hline Desloratadine & $10(10.9)$ & $3.8(1.9-7.3)$ \\
\hline Ebastine & $15(8.0)$ & $2.7(1.6-4.5)$ \\
\hline Fexofenadine & $1(1.8)$ & $0.5(0.1-4.0)$ \\
\hline Loratadine & $15(8.4)$ & $2.8(1.6-4.8)$ \\
\hline Mizolastine & $3(5.1)$ & $1.6(0.5-5.3)$ \\
\hline Rupatadine & $3(9.4)$ & $3.2(1.0-10.5)$ \\
\hline Terfenadine & $7(7.2)$ & $2.4(1.1-5.2)$ \\
\hline Levocetirizine & $4(16.7)$ & $6.2(2.1-18.1)$ \\
\hline
\end{tabular}

$\%$ Refers to the percent of the total number of reports for each particular drug. For all drugs, the total number of reports of heart rhythm disturbances since rupatadine was first marketed on 1 March 2003 and until the date of data collection for this study was 924 . The total number of reports for all adverse reactions in general in the corresponding period was 29,330 . For the antihistamines, the corresponding total number of reports of heart rhythm disturbances since they were first marketed were: astemizole (withdrawn on 1 April 2003), 2,237 (1 October 1985); azelastine, 2,583 (1 April 1993); cetirizine, 2,963 (1 November 1989); desloratadine, 959 (1 January 2003); ebastine, 2,958 (1 December 1989); fexofenadine, 1,764 (1 September 1998); loratadine, 2,958 (1 December 1989); mizolastine, 1,829 (1 March 1998); tefenadine, 3,146 (1 April 1985); and levocetirizine, 904 (1 April 2003). The total numbers of reports in the corresponding periods for all reactions were $72,027,79,890,93,621,30,445$, $93,458,55,584,93,458,58,024,100,877$, and 28,694 .

Reporting odds ratio (ROR) compared with estimates for other antihistamines. Data from the Spanish Pharmacovigilance System until December 2007.

cardiotoxicity; for the other drugs, the rates range from 0.4 cases per 100,000 patients treated per year for mizolastine to 3.1 for desloratadine, with overlapping confidence intervals.

\section{DISCUSSION}

We examined data from spontaneous reporting schemes to identify cases of heart rhythm disturbances in patients treated with rupatadine. The same adverse reactions have been observed for most of the other newer antihistamines and are well established for the older ones. The case of torsade de pointes included in this study has been described in detail elsewhere. ${ }^{8}$ Some of these adverse reactions are cause for concern because these drugs are commonly used for prolonged periods to treat a great number of patients with a wide range of conditions who are taking other medications at the same time. Such adverse reactions have prompted withdrawal of the antihistamines terfenadine and astemizole from the market. ${ }^{5,6}$

Although heart rhythm disturbances vary in their origin and severity, they can have similar consequences: blood flow to the brain is impaired, which can result in loss of consciousness (e.g., syncope) or even sudden death.

No causal relationship can be definitively established on a case-report basis. However, in all cases, the heart rhythm disturbances began after exposure to rupatadine and resolved when the drug was discontinued. In two cases, rupatadine was the only medication the patients were taking, and no other condition was diagnosed that could explain the heart rhythm disturbances. Because heart rhythm disturbances associated with antihistamines-particularly the most serious reactions- have a very low incidence, these rare but potentially fatal events can be identified only in a large population of patients with a broad range of conditions. In our series, one patient had a history of cardiovascular disease, and another was also taking sertraline, which may prolong the QT interval.

Heart rhythm disturbances have been reported for almost all antihistamines on the market. ${ }^{3-6}$ In the published data from clinical trials of rupatadine, however, no cardiotoxicity has been identified. ${ }^{9}$ In a "thorough QT/QTc" study conducted in 160 healthy volunteers, no significant increase in QT intervals was observed with doses of 10 and $100 \mathrm{mg},{ }^{10,11}$ even though peak plasma concentration and area under the curve increased and systemic clearance of the drug decreased after 7 days of once-daily treatment with rupatadine in healthy older volunteers compared with younger individuals. These data come from a small number of individuals and do not cover all of the clinical circumstances that 
Table 3 Rupatadine and heart rhythm disturbances

\begin{tabular}{|c|c|c|c|c|}
\hline Antihistamine & $\begin{array}{l}\text { Cases of heart rhythm } \\
\text { disturbances, } n\end{array}$ & $\mathrm{DDDs}^{\mathrm{a}}$ & Patients, $n$ & $\begin{array}{c}\text { Reporting rate per } 100,000 \\
\text { patients treated per year } \\
\text { (95\% confidence interval) }\end{array}$ \\
\hline Astemizole & 8 & $194,600,456$ & 532,787 & $1.5(0.6-3.0)$ \\
\hline Azelastine & 1 & $68,301,500$ & 186,999 & $0.5(0.0-3.0)$ \\
\hline Cetirizine & 8 & $513,350,785$ & $1,405,478$ & $0.6(0.2-1.1)$ \\
\hline Desloratadine & 10 & $115,705,740$ & 316,785 & $3.1(1.5-5.8)$ \\
\hline Ebastine & 15 & $645,620,000$ & $1,767,611$ & $0.8(0.5-1.4)$ \\
\hline Fexofenadine & 1 & $96,529,290$ & 264,283 & $0.4(0.0-2.1)$ \\
\hline Loratadine & 15 & $441,030,292$ & $1,207,475$ & $1.2(0.7-2.0)$ \\
\hline Mizolastine & 3 & $90,636,480$ & 248,149 & $1.2(0.2-3.5)$ \\
\hline Rupatadine & 3 & $53,650,180$ & 146,886 & $2.0(0.4-6.0)$ \\
\hline Terfenadine & 7 & $94,724,751$ & 259,342 & $2.7(1.1-5.6)$ \\
\hline Levocetirizine & 4 & $78,244,320$ & 214,221 & $1.9(0.5-4.8)$ \\
\hline
\end{tabular}

Reporting rate compared with that for the other antihistamines.

Data from the Spanish Pharmacovigilance System and from consumption through the Spanish National Health System until December 2007. Rupatadine was first marketed on 1 March 2003; astemizole on 1 October 1985 (withdrawn on 1 April 2003); azelastine on 1 April 1993; cetirizine on 1 November 1989; desloratadine on 1 January 2003; ebastine on 1 December 1989; fexofenadine on 1 September 1998; loratadine on 1 December 1989; mizolastine on 1 March 1998 ; terfenadine on 1 April 1985 ; levocetirizine on April 2003. All searches were conducted between the date of first marketed until December 12th, except for astemizole, which was conducted until date of withdrawal (1 April 2003 ); the last item is stated in the Methods section.

DDDs, defined daily doses.

ahttp://www.whocc.no/atcddd.

can account for higher susceptibility to arrhythmias, including hypokalemia, preexisting cardiac conditions, and coadministration of drugs that prolong the QT interval or inhibit metabolism. On the other hand, experiments with cloned human myocytes have shown that rupatadine can cause concentration-, time-, and voltage-dependent blockade of hKv1.5 channels involved in the duration of the action potentials in the human heart. ${ }^{12}$ The peak plasma concentration in healthy volunteers after oral administration of $20 \mathrm{mg} /$ day of rupatadine (the highest therapeutic dose) was $5.5 \mathrm{nmol} / \mathrm{l}^{13}$ This concentration is $<1 / 400$ of the $K_{\mathrm{D}}$ for hKv1.5 blockade $(2,400 \mathrm{nmol} / \mathrm{l})$. As $K_{\mathrm{D}}$ is the concentration that produces $50 \%$ of the maximum inhibitory effect and the inhibitory effect is evident at concentrations of $10 \mathrm{nmol} / \mathrm{l}$, some degree of blockade might be expected for concentrations between 5.5 and $10 \mathrm{nmol} / \mathrm{l}$. This range of concentrations is close to those reached with therapeutic doses of rupatadine and could be more readily reached in patients taking cytochrome $\mathrm{P} 4503 \mathrm{~A} 4$ inhibitors at the same time. It is also possible that a mutated $\mathrm{hKv} 1.5$ was more sensitive to the drug than expected. ${ }^{14}$

Despite underreporting, ${ }^{15}$ the data from Spain show a statistical association between rupatadine use and heart rhythm disturbances. The estimated association is similar to those for antihistamines known to induce such reactions. Because this is the first reported case series of heart rhythm disturbances thought to be induced by rupatadine, it is unlikely that a "notoriety bias" accounts for this association. The absence of such bias is supported by the fact that both the reporting odds ratio and the reporting rate point to a risk similar to those associated with other antihistamines. It is possible that some patients who had problems with other antihistamines or who were thought to be at risk of developing cardiotoxicity were switched to rupatadine (channeling). This would account for a higher estimate, but it would not indicate an absence of risk.
Torsade de pointes, the most serious antihistamine-induced heart rhythm disturbance, is thought to result from prolonged ventricular repolarization. Patients with this condition show a marked prolongation of the QT interval on electrocardiograms. ${ }^{16}$ Antihistamines that do not block cardiac voltage-gated potassium currents related to repolarization, particularly the rapid component of the delayed rectifier potassium current, would not induce torsade. Nevertheless, under certain circumstances, including drug-drug interactions and kidney or liver impairment, rupatadine may reach plasma concentrations far above the therapeutic range that can block those currents. Other mechanisms by which antihistamines could induce severe heart rhythm disturbances have been proposed, including muscarinic receptor inhibition in the heart, ${ }^{17}$ calcium interference, ${ }^{18}$ and histamine release. ${ }^{19}$ For example, desloratadine, the main metabolite of rupatadine, has been shown to release histamine from cardiac mast cells. ${ }^{20}$

The low incidence of drug-induced arrhythmia makes collaborations between countries, such as the one established for this study, particularly useful. As a system for "signal generation," the spontaneous reporting is considered key to the early identification of proarrhythmic newly marketed drugs. ${ }^{21}$ The prevalence of allergic conditions has been increasing, and they are being treated for longer. Because $\mathrm{H} 1$ antihistamines are first-line medications for these conditions, they are among the most frequently prescribed drugs worldwide. It is a matter of debate whether arrhythmias are a class effect. Our results support the idea that antihistamines as a group are associated with an increased risk of heart rhythm disturbances. Thus, a statement should be included in the summary of product characteristics to indicate a possible association between rupatadine and cardiotoxicity. Physicians should be aware of the potential cardiotoxic effect of antihistamines and avoid prescribing them for patients who are prone 
to these rare but potentially life-threatening heart rhythm disturbances. These include patients with hereditary long-QT syndrome, who are particularly sensitive to any factor that further prolongs the QT interval; patients taking cytochrome P450 3A4 inhibitors; and patients with kidney or liver impairment.

\section{METHODS}

The Spanish and Portuguese pharmacovigilance system databases used in this study include all adverse drug reactions reported to regional pharmacovigilance centers and coordinator centers in both countries. Physicians and pharmacists submit spontaneous reports of suspected adverse drug reactions. In particular, the centers request reports of severe events and of events associated with the use of recently marketed drugs. Ad hoc committees evaluate reports using an algorithm to determine whether a causal relationship exists. Reports are included in the databases regardless of causality and severity. Until July 2007, adverse drug reactions were coded according to the World Health Organization Adverse Reactions Terminology dictionary; ${ }^{22}$ since then, the MedDRA dictionary has been used. ${ }^{23}$

We used a case/noncase approach to assess the strength of the association between rupatadine exposure and cardiotoxicity. ${ }^{24}$ We took only the data from the Spanish database, as it included enough cases of interest. Cases were defined as reports of heart rhythm disturbances; noncases were defined as reports of all reactions other than heart rhythm disturbances. Exposure was defined as the mention of rupatadine in a report, whether or not it was suspected of causing the reaction. The search extended from the date each antihistamine came onto the market until 12 December 2007, except in the case of astemizole, which was withdrawn from the Spanish market in 2003.

The association between heart rhythm disturbances and rupatadine was estimated by calculating the reporting odds ratio with a 95\% confidence interval. ${ }^{25-27}$ The same ratio was calculated for other antihistamines on the market to compare the risks of heart rhythm disturbances. Drugs combining an antihistamine with one or more additional drugs (e.g., ebastine plus pseudoephedrine) were excluded from the study.

The reporting rate in Spain was calculated for all drugs studied as the number of reported cases divided by the number of person-years of treatment. To estimate the number of patients exposed, we used drug sales data from the Spanish National Health System extracted from the Especialidades Consumo de Medicamentos database of the Ministry of Health. This database contains information on community drug consumption throughout the Spanish National Health System, which covers $99 \%$ of the population. Drug consumption data were converted into defined daily doses and then into number of person-years of treatment. The reporting rate was estimated on the assumption that the exposed population was large and the number of cases few. On this assumption, reporting of suspected adverse reactions is expected to follow a Poisson distribution, and confidence limits can be calculated on the basis of the relation between Poisson and $\chi^{2}$ distribution. ${ }^{28,29}$

\section{ACKNOWLEDGMENTS}

Instituto de Farmacoepidemiología (Universidad de Valladolid) receives funds for pharmacovigilance activities from the Direccion General de Salud Pública e Investigación, Desarrollo e Innovación. Consejería de Sanidad. Junta de Castilla y León.

\section{CONFLICT OF INTEREST}

The authors declared no conflict of interest.

(C) 2009 American Society for Clinical Pharmacology and Therapeutics

1. Martindale: The Complete Drug Reference Accessed Through MedicinesComplete <http://www.medicinescomplete.com/mc/martindale/ current/advanced.htm?a=rupatadine\&ss=all $>$. Accessed March 2007.

2. Merlos, M. et al. Rupatadine, a new potent, orally active dual antagonist of histamine and platelet-activating factor (PAF). J. Pharmacol. Exp. Ther. $\mathbf{2 8 0}$ 114-121 (1997)
3. Lindquist, M. \& Edwards, I.R. Risks of non-sedating antihistamines. Lancet 349, 1322 (1997).

4. de Abajo, F.J. \& Rodríguez, L.A. Risk of ventricular arrhythmias associated with nonsedating antihistamine drugs. Br. J. Clin. Pharmacol. 47, 307-313 (1999).

5. Woosley, R.L. Cardiac actions of antihistamines. Annu. Rev. Pharmacol. Toxicol. 36, 233-252 (1996).

6. Monahan, B.P., Ferguson, C.L., Killeavy, E.S., Lloyd, B.K., Troy, J. \& Cantilena, L.R. Jr. Torsades de pointes occurring in association with terfenadine use. JAMA 264, 2788-2790 (1990).

7. Handley, D.A., Magnetti, A. \& Higgins, A.J. Therapeutic advantages of third generation antihistamines. Expert Opin. Investig. Drugs 7, 1045-1054 (1998).

8. Nombela-Franco, L., Ruiz-Antoran, B., Toquero-Ramos, J. \& Silva-Melchor, L. Torsades de pointes associated with rupatadine. Rev. Esp. Cardiol. 61, 328-329 (2008).

9. Keam, S.J. \& Plosker, G.L. Rupatadine: a review of its use in management of allergic disorders. Drugs 67, 457-474 (2007).

10. The clinical evaluation of QT/QTc interval prolongation and proarrhythmic potential for non-antiarrhythmic drugs. ICH Harmonised Tripartite Guideline. Current step 4 version. International Conference for Harmonisation of Technical Requirements for Registration of Pharmaceuticals for Human Use, 12 May 2005 [online] <http//www.ich.org >

11. Donado, E. et al. Cardiac safety of rupatadine according to the new ICH guideline: a "thorough QT/QTc study". XXV Congress of the European Academy of Allergology and Clinical Immunology, Vienna, Austria, 10-14 June 2006. Abstract 760.

12. Caballero, R., Valenzuela, C., Longobardo, M., Tamargo, J. \& Delpón, E. Effects of rupatadine, a new dual antagonist of histamine and platelet-activating factor receptors, on human cardiac Kv1.5 channels. Br. J. Pharmacol. 128, 1071-1081 (1999).

13. García Rafanell, J. Rupatadine fumarate: antiallergic histamine and PAF antagonist. Drugs Future 21, 1032-1036 (1996).

14. Fitzgerald, P.T. \& Ackerman, M.J. Drug-induced torsades de pointes: the evolving role of pharmacogenetics. Heart Rhythm 2 (2 suppl.), S30-S37 (2005)

15. Alvarez-Requejo, A., Carvajal, A., Bégaud, B., Moride, Y., Vega, T. \& Arias, L.H. Under-reporting of adverse drug reactions. Estimate based on a spontaneous reporting scheme and a sentinel system. Eur. J. Clin. Pharmacol. 54, 483-488 (1998)

16. Tamargo, J. Drug-induced torsade de pointes: from molecular biology to bedside. Jpn. J. Pharmacol. 83, 1-19 (2000).

17. Liu, $H_{\text {., }}$ Zheng, Q. \& Farley, J.M. Antimuscarinic actions of antihistamines on the heart. J. Biomed. Sci. 13, 395-401 (2006).

18. Hove-Madsen, L. et al. The proarrhythmic antihistaminic drug terfenadine increases spontaneous calcium release in human atrial myocytes. Eur. J. Pharmacol. 553, 215-221 (2006).

19. Llenas, J., Cardelús, I., Heredia, A., de Mora, F. \& Gristwood, R.W. Cardiotoxicity of histamine and the possible role of histamine in the arrhythmogenesis produced by certain antihistamines. Drug Saf. 21 (suppl. 1), 33-38; discussion 81-87 (1999).

20. Heredia, A., Beleta, J. \& Llenas, J. Terfenadine and the main metabolite of loratadine release histamine from cardiac mast cells: possible cardiotoxic consequences. Ann. Allergy Asthma Immunol. 78, 142 (1997).

21. Darpö, B. Detection and reporting of drug-induced proarrhythmias: room for improvement. Europace 9 (suppl. 4), iv23-iv36 (2007).

22. World Health Organization. International Monitoring of Adverse Reactions to Drugs: Adverse Reaction Terminology (World Health Organization Collaborating Centre for International Drug Monitoring, Uppsala, Sweden, 1992).

23. Brown, E.G., Wood, L. \& Wood, S. The medical dictionary for regulatory activities (MedDRA). Drug Saf. 20, 109-117 (1999).

24. Moore, N. et al. Reports of hypoglycaemia associated with the use of ACE inhibitors and other drugs: a case/non-case study in the French pharmacovigilance system database. Br.J. Clin. Pharmacol. 44, 513-518 (1997).

25. Evans, S.J. Pharmacovigilance: a science or fielding emergencies? Stat. Med. 19, 3199-3209 (2000)

26. Rothman, K.J., Lanes, S. \& Sacks, S.T. The reporting odds ratio and its advantages over proportional reporting ratio. Pharmacoepidemiol. Drug Saf. 13, 519-523 (2004).

27. Miettinen, O.S. \& Wang, J.D. An alternative to the proportionate mortality ratio. Am. J. Epidemiol. 114, 144-148 (1981).

28. Ulm, K. A simple method to calculate the confidence interval of a standardized mortality ratio (SMR). Am. J. Epidemiol. 131, 373-375 (1990).

29. Dobson, A.J., Kuulasmaa, K., Eberle, E. \& Scherer, J. Confidence intervals for weighted sums of Poisson parameters. Stat. Med. 10, 457-462 (1991). 